\title{
Factors Associated With Neonatal Pneumonia and its Mortality in India: A Systematic Review and Meta-Analysis
}

\author{
N Sreekumaran Nair, ${ }^{1}$ Leslie Edward Lewis, ${ }^{2}$ ViJay Shree Dhyani, ${ }^{1}$ Shruti Murthy, ${ }^{1}$ Myron Godinho, ${ }^{1}$ \\ Theophilus Lakiang, ${ }^{\mathbf{1}}$ BHUMika T Venkatesh ${ }^{\mathbf{1}}$ \\ From ${ }^{1}$ Department of Statistics, Public Health Evidence South Asia (PHESA); and ${ }^{2}$ Department of Pediatrics, Kasturba Medical \\ College Hospital; Manipal Academy of Higher Education, Manipal, Karnataka. \\ Correspondence to: Dr Bhumika T Venkatesh, Room no. 35, Public Health Evidence South Asia (PHESA), Prasanna School of Public \\ Health, Manipal Academy of Higher Education, Madhav Nagar, Manipal, Karnataka.meetbhumika123@gmail.com \\ Received: May 19, 2020; Initial review: June 29, 2020; Accepted: March 13, 2021.
}

Background: Neonatal pneumonia remains a significant contributor to infant mortality in India and responsible for increased prevalence of infant deaths globally. Objective: To identify risk factors associated with neonatal pneumonia and its mortality in India. Study design: A systematic review was conducted including both analytic study designs and descriptive study designs, which reported a quantitative analysis of factors associated with all the three types of pneumonia among neonates. The search was conducted from August to December, 2016 on the following databases; CINAHL, EMBASE, Ovid MEDLINE, PubMed, ProQuest, SCOPUS, Web of Science, WHO IMSEAR and IndMED. The search was restricted to Indian setting. Participants: The population of interest was neonates. Outcomes: The outcome measures included risk factors for incidences and mortality predictors of neonatal pneumonia. These could be related to neonate, maternal and pregnancy, caregiver, family, environment, healthcare system, iatrogenic and others. Results: A total of three studies were included. For risk factors, two studies on ventilator-associated pneumonia were included with 194 neonates; whereas for mortality predictors, only one study with 150 neonates diagnosed with pneumonia was included. 11 risk factors were identified from two studies: duration of mechanical ventilation, postnatal age, birth weight, prematurity, sex of the neonate, length of stay in NICU, primary diagnosis, gestational age, number of re-intubation, birth asphyxia, and use of nasogastric tube. Metaanalysis with random-effects model was possible only for prematurity $(<37$ week) and very low birth weight $(<1500 \mathrm{~g})$ and very low birth weight was found to be significant (OR $5.61 ; 95 \% \mathrm{Cl} 1.76,17.90)$. A single study was included on predictors of mortality. Mean alveolar arterial oxygen gradient (AaDO2) $>250 \mathrm{~mm} \mathrm{Hg}$ was found to be the single most significant predictor of mortality due to pneumonia in neonates. Conclusion: The study found scant evidence from India on risk factors of neonatal pneumonia other than ventilator-associated pneumonia.

Keywords: Alveolar-arterial oxygen gradient, Respiratory distress, Risk factors, Ventilator-associated pneumonia.

Protocol registration: PROSPERO 2016 CRD42016044019 (risk factors); PROSPERO 2016 CRD42016045398 (mortality)

$\mathrm{N}$ eonatal pneumonia accounts for $6.1 \%$ of total global neonatal mortality whereas it contributes $5.1 \%$ to neonatal mortality in India and $5.6 \%$ in South Asia [1]. There is no international consensus regarding definition, diagnostic criteria and management of pneumonia among neonates [2,3]. National nosocomial infections surveillance (NNIS) 1996 and original Centers for Disease Control (CDC) guidelines (pediatric modification) are commonly followed for diagnosis of neonatal pneumonia.

It has been observed that poverty, limited healthcare accessibility, and improper child-rearing practices are some of the risk factors for pneumonia in young children [4]. Other factors related to development of pneumonia, particularly in India, are financial status, malnutrition, poor immunization status, and household air pollution [5]. In South East Asia, poor prenatal care, home delivery, fever at birth, maternal urinary tract infections, prolonged rupture of membrane were found as notable risk factors of neonatal pneumonia $[6,7]$.
There is scanty information available on neonatal pneumonia from India. Identification and elimination of risk factors associated with neonatal pneumonia is imperative to reduce its high prevalence and associated mortality, and implementing appropriate interventions to improve neonatal survival. With this review we intended to identify risk factors and mortality predictors associated with neonatal pneumonia in the Indian context.

\section{METHODS}

Protocol for these systematic reviews were registered with PROSPERO [8,9] and published as separate publications $[10,11]$ where methodology is described in detail. Ethical clearance was obtained from institutional ethics committee of the host institution.

Studies reporting all types of neonatal pneumonia published in English language in journals, irrespective of peer reviewed or not were eligible for inclusion. Studies on neonatal sepsis were also searched to verify the presence or 
absence of a 'pneumonia' subgroup as pneumonia is usually considered under the umbrella of neonatal sepsis. To be eligible for inclusion, these articles had to mention the outcomes specifically for neonatal pneumonia.

Both analytic study designs (case-control studies, cohort studies, cross-sectional studies) and descriptive study designs (case series, cross-sectional studies) which report a quantitative analysis of factors associated with all the three types of pneumonia among neonates were eligible for inclusion. Letters, editorials, commentaries, reviews, meta-analysis, qualitative research, conference papers and reports were excluded.

Neonates diagnosed with any form of pneumonia including community acquired pneumonia, congenital pneumonia and hospital acquired pneumonia (ventilator associated pneumonia) were included. The outcome measures included risk factors for neonatal pneumonia and its mortality. These could be related to neonate, maternal and pregnancy, caregiver, family, environment, healthcare system, iatrogenic and others.

Search methods: Articles were identified from nine databases (CINAHL, EMBASE, Ovid MEDLINE, ProQuest, PubMed, SCOPUS, WHO IMSEAR, Web of Science and IndMED) and government websites without time restriction. A separate search was undertaken to identify risk factors and mortality predictors associated with neonatal pneumonia. Detailed search terms and strategy for PubMed for both the outcomes has been provided in Web Appendix 1. The search on all the databases was conducted from August to December, 2016. Some of the search terms included were: "Risk factor" OR "determinant" OR "risk" OR "predictor" AND "Mortality" OR "fatal" OR "case fatality" OR "case fatality rate" AND "Neonate" OR "childhood" OR "neonatal” OR "newborn" AND "Pneumonia" OR "hospital acquired pneumonia") OR “community-acquired pneumonia" OR "ventilator associated pneumonia" OR "early onset pneumonia" OR "late onset pneumonia." Additionally grey literature search and snowballing were also conducted to find out potentially relevant studies. The authors were contacted in an attempt to retrieve missing information on important methodological aspects or outcomes measures.

Data extraction and quality assessment: Considering inclusion and exclusion criteria, three review authors (SM, MG, and TL) worked in two teams to screen, extract data and quality assessment of identified literature. The consensus for any discrepancies were sought through discussion with senior reviewers (NSN, LL, and BTV). APreferred Reporting Items for Systematic Reviews and Meta-Analyses (PRISMA) chart was generated to summarize the study selection process. Characteristics were summarized and results were reported using tables and accompanied by a descriptive summary that compared and evaluated the methods and results of included studies. The results of the search were managed and screened using Endnote (v. x7). Microsoft Excel 2007 was utilized for data extraction.

Statistical analysis: Depending on methodological heterogeneity, a random-effects model was used. The summary measures were pooled based on study design. A Forest plot was generated and pooled estimates were reported with 95\% CIs. Based on the availability of data, a subgroup analysis was also planned a priori with respect to study design, type of neonatal pneumonia, study setting, and onset of pneumonia. However, the subgroup analysis was not possible due to non-availability of relevant data. For metaanalysis, data were available only from two studies on VAP (ventilator-associated pneu-monia) and meta-analysis was possible only for two factors i.e., very low birth weight and prematurity. Depending on data availability, a sensitivity analysis and meta-regression was planned but could not be performed due to limited data. Reporting bias could also not be assessed as included studies were less than 10 .

The reporting has been done in accordance with the Preferred Reporting Items for Systematic reviews and MetaAnalysis (PRISMA) guidelines [12] and the Meta-analysis of Observational Studies in Epidemiology (MOOSE) guidelines [13]. Quality assessment was done at the study level using the modified Quality Assessment Tool for Systematic Reviews of Observational Studies (QATSO) tool [14]. STATA(v.13) was used to perform statistical analyses.

\section{RESULTS}

A total of 8754 citations were subjected to title screening, and finally two articles were found to be eligible and were included for the meta-analysis (Fig. 1). For mortality predictors of neonatal pneumonia, a total of 6,955 citations were identified, of which, 303 articles were screened for full text and only one article was eligible for inclusion (Fig. 2). Meta-analysis was not possible as there was only one eligible study.

For risk factors, two studies $[15,16]$ were included with data from a total of 194 neonates. For mortality predictors, only one study [17] with 150 neonates was included. Table I lists the characteristics of included studies [18].

Both studies used (NNIS) 1996 guidelines in conjunction with pediatric modification of the original center for disease control guidelines. [15,16]. Both studies (risk factors) found Klebsiella species as the most predominantly isolated organism from the endotracheal aspirate of neonates with ventilator associated pneumonia. None of the studies reported the socio-demographic characteristics of neonates with or without ventilator associated pneumonia. Web Table 


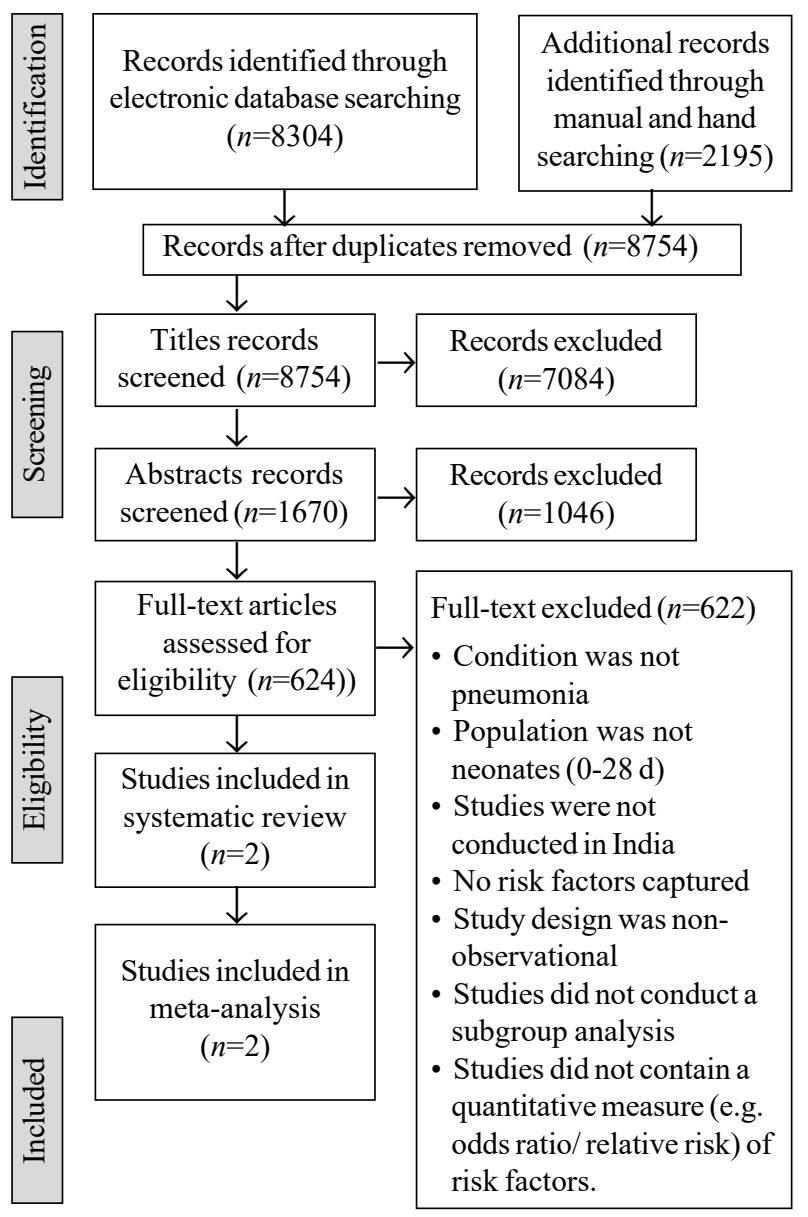

Fig. 1 PRISMA flow chart depicting the study selection process for risk factors of neonatal pneumonia.

I describes in detail diagnostic criteria used for ventilator associated pneumonia $[15,16]$ and neonatal pneumonia [17]. The study on mortality predictors did not specify the guideline followed for diagnosis of neonatal pneumonia, the authors reported the use of the National Neonatology Forum (NNF) to diagnose 'respiratory problems'. In both the studies $[15,16]$ no primary criteria for mechanical ventilation was provided in the methodology; however, in one of the study results indicated four conditions for mechanical ventilation namely pneumonia, apnea, poor respiratory effort and Hyaline Membrane Disease [15].

In total, 11 risk factors were identified from two studies and six of them were common across both studies. Table I provides a risk factor profile of the included studies [15, 16]. A random effects model was used for the meta-analysis. Metaanalysis was carried out for only two factors from two studies namely very low birthweight (VLBW) and prematurity. In both the included studies, a significant association was found

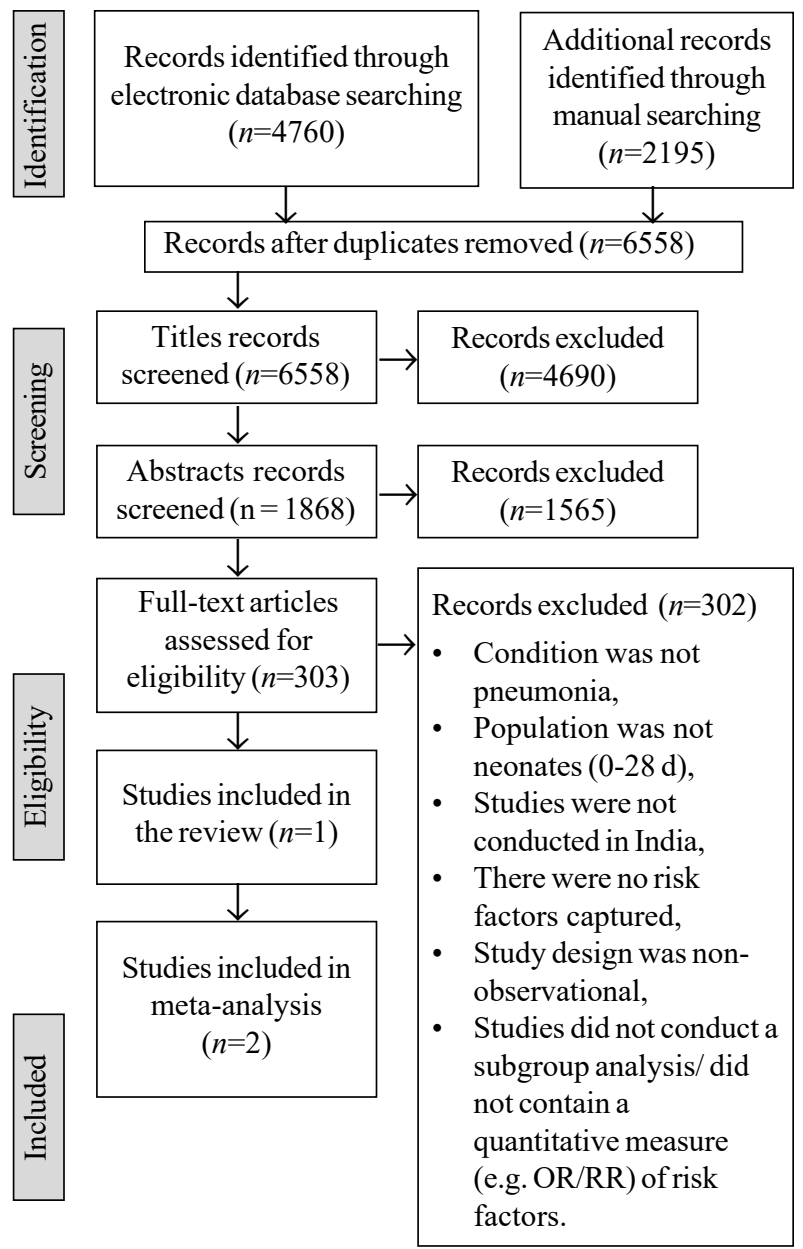

Fig. 2 PRISMA flowchart depicting the study selection process for factors associated with mortality.

between development of ventilator associated pneumonia and duration of mechanical ventilation and number of reintubations; however, there was missing data and attempts at reaching the authors were unsuccessful, therefore a metaanalysis could not be performed for these factors.

Pooled OR for very low birth weight from random effects meta-analysis of two studies [15,16] is depicted in Fig. 3. The forest plot show that neonates with VLBW $(<1500 \mathrm{~g})$ were more likely to develop ventilator associated pneumonia compared to neonates who were normal to low birthweight (OR 5.61;95\% CI 1.76, 17.90). Very low birth weight was found to be significant risk factor for development of ventilator associated pneumonia. Pooled OR for prematurity is depicted in Fig. $4[15,16]$. The Forest plot shows that neonates with estimated gestational age $<37$ week or premature neonates were more likely to develop ventilator associated pneumonia compared to term neonates (OR 2.76; 95\% CI $0.98,7.73)$. 
Table I Characteristics of Included Studies

\begin{tabular}{|c|c|c|c|c|c|}
\hline Study, year & Study design & Location \& setting & Study duration & Outcome & Risk factors and mortality predictors \\
\hline $\begin{array}{l}\text { Tripathi, } \\
2010[15]\end{array}$ & $\begin{array}{l}\text { Cross-sectional } \\
\text { study }\end{array}$ & $\begin{array}{l}\text { Lucknow, Uttar Pradesh } \\
\text { NICU, tertiary care teaching } \\
\text { hospital }\end{array}$ & $1 \mathrm{y}$ & VAP & $\begin{array}{l}\text { Risk factors } \\
\text { Duration of mechanical ventilation (h) } \\
\text { Postnatal age (admission) } \\
\text { Birth weight } \\
\text { Prematurity } \\
\text { Sex of the neonate } \\
\text { Length of NICU stay } \\
\text { Primary diagnosis } \\
\text { Gestational age } \\
\text { Number of re-intubation }\end{array}$ \\
\hline $\begin{array}{l}\text { Mir, } \\
2015[16]\end{array}$ & $\begin{array}{l}\text { Cross-sectional } \\
\text { study }\end{array}$ & $\begin{array}{l}\text { Srinagar, Kashmir } \\
\text { NICU, teaching, } \\
\text { referral hospital }\end{array}$ & $10 \mathrm{mo}$ & VAP & $\begin{array}{l}\text { Risk factors } \\
\text { Duration of mechanical ventilation (h) } \\
\text { Postnatal age (initiation) } \\
\text { Birth weight } \\
\text { Prematurity } \\
\text { Sex of the neonate } \\
\text { Length of NICU stay } \\
\text { Primary diagnosis } \\
\text { Number of re-intubation } \\
\text { Birth asphyxia } \\
\text { Use of Nasogastric tube }\end{array}$ \\
\hline $\begin{array}{l}\text { Mathur, } \\
2002 \text { [17] }\end{array}$ & $\begin{array}{l}\text { Cross-sectional } \\
\text { study }\end{array}$ & $\begin{array}{l}\text { New Delhi } \\
\text { Referral neonatal } \\
\text { unit of a teaching } \\
\text { hospital }\end{array}$ & Not specified & NP & $\begin{array}{l}\text { Mortality predictors } \\
\text { AaDO2 gradient }>250 \mathrm{~mm} \mathrm{Hg} \\
\text { Birth weight }<2000 \mathrm{~g}, \\
\text { Gestation }<34 \text { weeks } \\
\text { Age at presentation } \\
\text { Lethargy } \\
\text { Absent neonatal reflexes, } \\
\text { Shock } \\
\text { Silverman score }(4 \text { to } 6 \text { ), } \\
\text { FiO2 }>40 \% \text { pH }<7.2 \text {, base excess }>-10 \\
\text { Positive blood culture } \\
\text { CRP positive mean arterial alveolar } \\
\text { tension ratio (a/A ratio) }<0.25 \\
\text { positive ventilatory support }\end{array}$ \\
\hline
\end{tabular}

NICU-newborn intensive care unit, VAP-ventilator-associated pneumonia, NP-neonatal pneumonia.

Only one study [17] reported mortality predictors to neonatal pneumonia. The authors did not specify the list of independent and confounding variables considered as predictors for fatality due to pneumonia in neonates. However, they provided only $P$ values for significant predictors which they considered for multiple logistic regression. These predictors included: $<$ birthweight $2000 \mathrm{~g}$, gestation $<34$ week, age at presentation, lethargy, absent neonatal reflexes, shock, Silverman score (4 to 6), $\mathrm{FiO}>40 \%$, $\mathrm{pH}<7.2$, base excess $>-10$, positive blood culture, $\mathrm{C}$-reactive protein (CRP) positive, mean alveolar arterial oxygen gradient $(\mathrm{AaDO} 2)>250 \mathrm{~mm} \mathrm{Hg}$, mean arterial alveolar tension ratio (a/ A ratio) $<0.25$ and positive ventilatory support. The authors found only $\mathrm{AaDO} 2$ gradient $>250 \mathrm{mmHg}$ as a significant predictor of mortality due to pneumonia with respiratory distress in neonates (OR 71.1; 95\% CI 1.1,4395).

Publication bias could not be assessed as there were fewer than 10 studies. Web Table II depicts quality assessment of studies using the QATSO Tool [14]. Four of the five items on the scale were used to assess $(i)$ External validity, (ii) Reporting, (iii) Bias and (iv) Confounding. However, no scoring was done. For studies on risk factors, the measurement of pneumonia was only found to be objective in one study [15] i.e., clinical records or laboratory tests. Neither study reported any response rate. Regarding the control of confounding factors when analyzing associations, only one study partially accounted for this [15], while the other did not report adequately on the handling of variables during the analysis [16]. 


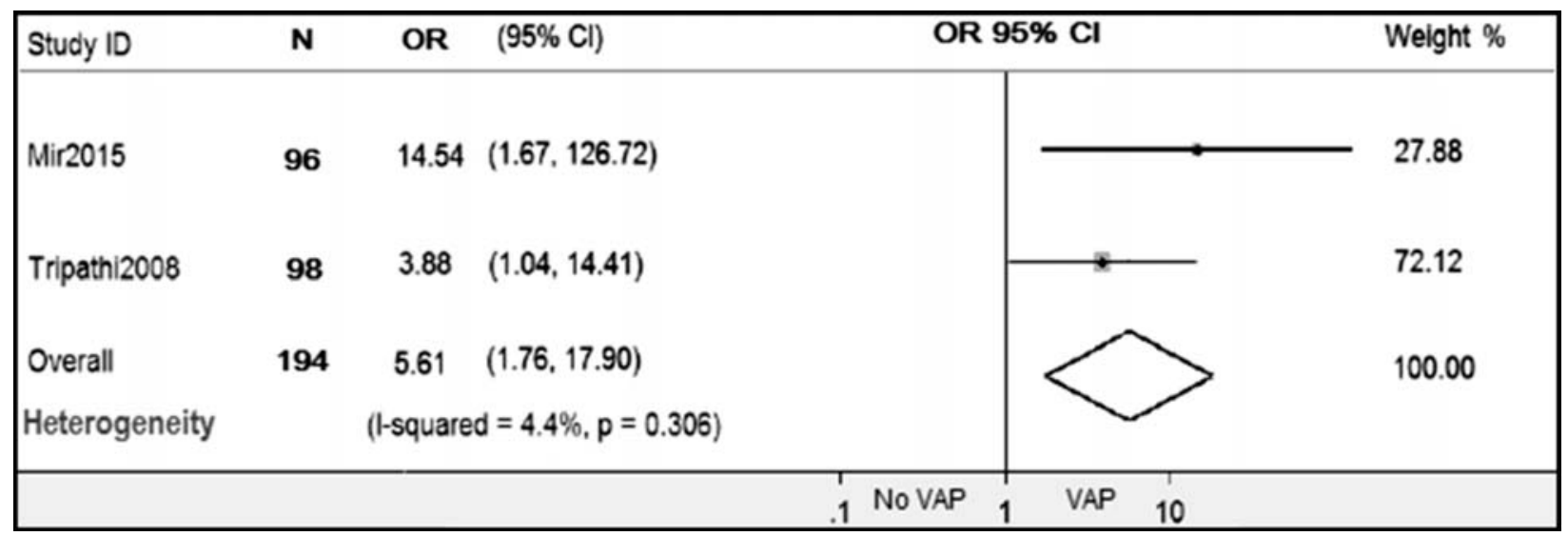

Fig. 3 Forest plot showing the effect of very low birthweight on ventilator-associated pneumonia.

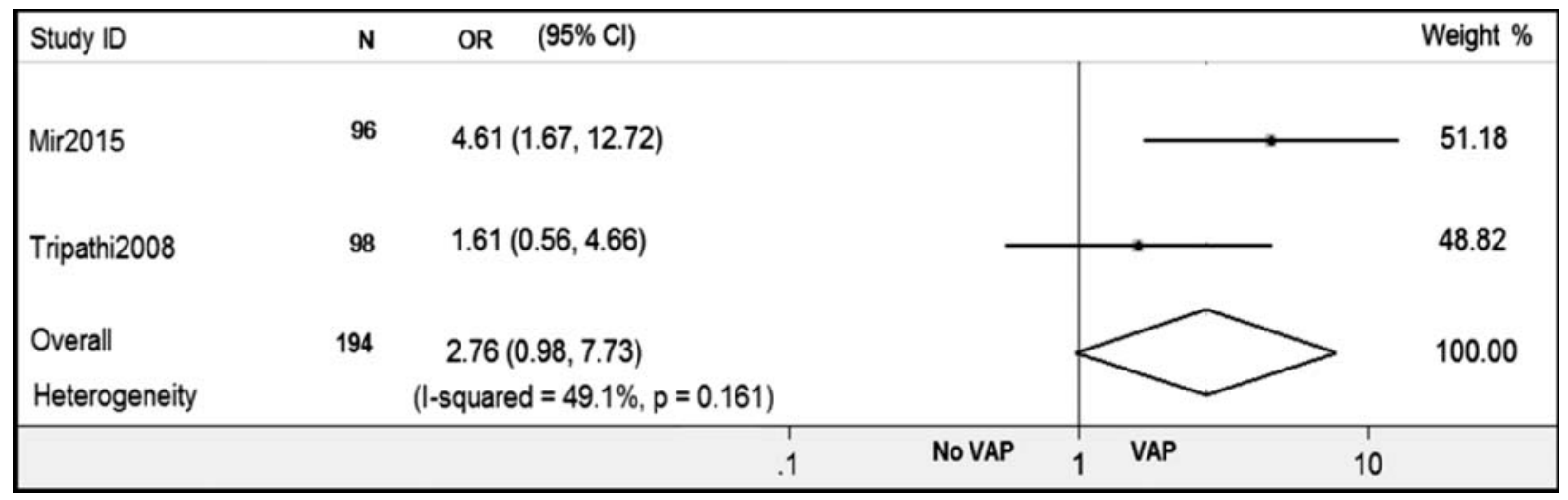

Fig. 4 Forest plot showing the effect of prematurity on ventilator-associated pneumonia.

For study on mortality predictors [17] dose response relationship could not be determined. The odds ratio in the study was very large with large confidence intervals (71.1; $95 \% \mathrm{CI} 1.1,4395)$. No event rates were reported for both the groups. Discrepancies exist in the numbers of participants included in the study. The authors mention the presence of two groups: respiratory distress with pneumonia and respiratory distress without pneumonia. However, information on socio-demographic characteristics, clinical and other important exposure and confounding information for the two groups was missing.

\section{DISCUSSION}

We conducted a series of systematic reviews to determine the risk factors associated with development of neonatal pneumonia and its mortality predictors in India. Literature is widely available on pneumonia in general and on neonatal sepsis. However there was near absence of data on neonatal pneumonia particularly with respect to its risk factors and mortality predictors in India. Only two studies were included for risk factors and only one study on mortality predictors of neonatal pneumonia. Meta-analysis for prematurity and low birth weight was carried out and low birth weight was found to be significant for the occurrence of neonatal pneumonia. Only alveolar-arterial oxygen gradient $(\mathrm{AaDO} 2)>250 \mathrm{~mm} \mathrm{Hg}$ was found as a significant predictor of mortality due to pneumonia with respiratory distress in neonates in present review.

To the best of our knowledge this systematic review is the first in India studying factors associated with pneumonia and its mortality in neonates. A rigorous effort was made to search the relevant studies without time restriction in Indian context by means of conducting search on nine electronic databases, hand searching, grey literature, by contacting authors and in consultation with clinical experts to include every possible study. Screening and data extraction was carried out independently by two authors and discrepancies were resolved by mutual discussion and by getting experts opinion. 
Considering the limited evidence in this review, studies on neonatal sepsis were included up to full text screening to verify the presence or absence of a subgroup for pneumonia but no clear underlying etiology as risk factor for neonatal pneumonia was mentioned in these studies. Consequently we have excluded studies where pneumonia was part of the condition but further description for neonatal pneumonia was not given separately.

Another limitation of the review was the lack of response from authors of studies of neonatal sepsis that did not explicitly provide data on the pneumonia component of their sepsis cases. Due to lack of data in the papers, meta-analysis for all the identified risk factors was not possible. Results from our study on risk factors pertain only to cases of ventilator associated pneumonia, which is a subgroup of neonatal pneumonia, and therefore, the findings could not be extrapolated to all the cases of neonatal pneumonia.

The major limitation for mortality predictors of neonatal pneumonia was that we found limited evidence from a single study that was not sufficient to conclude despite the comprehensiveness of our search. One of the potential limitation could be the language as we have restricted the search only to articles published in English. Nonetheless, we might have not missed any relevant studies on neonatal pneumonia as scientific literature in India is mostly published in English.

Both the studies $[15,16]$ in our review investigated ventilator-associated pneumonia in neonates that required mechanical ventilation for 48 hour or more as observed in other studies [19-23]. In our review the incidence of ventilator associated pneumonia ranged from 22 to 68 cases per 1000 MV days where as in another study from China it was 27.33 per 1,000 ventilator-days [21]. In contrast, in a study from USA, VAP rates were as low as 6.5 and 4 per 1000 ventilator days for patients with EGA $<28$ week and EGA $>28$ week, respectively [22]. Both the included studies $[15,16]$ found Klebsiella species as the most predominantly isolated organism from the endotracheal aspirate of neonates with VAP. Similarly in studies from Egypt [23] and Western India [24], K. pneumoniae was also found to be the most common organism.

High AaDO2 was found as a significant predictor of mortality due to pneumonia. Similarly, in a study from Bangladesh, high AaDO2 was one of the factors significantly associated with change in antibiotics due to the worsening condition of the neonates diagnosed with pneumonia [25]. However, they did not specify the limit to describe AaDO2 as high whereas AaDO2 $>250 \mathrm{~mm} \mathrm{Hg}$ was considered as high in the included study [17]. In a multivariate logistic regression, VAP was the single most important factor found to be significantly associated with mortality, whereas marginally significant association was found with presence of an arterial catheter [22].

In contrast to our meta-analysis findings and few other studies [19,20,22,26-28], there is one study [29] where the occurrence of VAP was not associated with low birth-weight $(<1500 \mathrm{~g})$. Results from meta-analysis of two studies [15,16] found birth-weight of $<1500 \mathrm{~g}$ as a significant risk factor to develop VAP(OR 5.61; 95\% CI 1.76, 17.90) Our meta-analysis findings are comparable to a study from China where low birth weight and premature infants had more chances of developing VAP[28].

Differences in birth weight were observed amongst different studies when it comes to defining weight at birth as low. One study in our review [15] defined VLBW as less than $1500 \mathrm{~g}$. The other study [16] defined it as in between 1000 to $1500 \mathrm{~g}$ and excluded extremely low birth weight babies less than $1000 \mathrm{~g}$ weight. However, a study from Thailand reported a neonatal birth weight less than $750 \mathrm{~g}$ as an independent risk factor for VAP [19] and another study established that VAP rates were high among extremely preterm neonates but birth weight was specified as $\leq 2000 \mathrm{~g}$ [22]. A retrospective observational study conducted in Taiwan found that higher gestational age and weight at birth were significantly associated in bringing down the VAP occurrence [20].

Like other studies [27-29], duration of NICU stay and MV were found as the risk factors but due to lack of data, metaanalysis for these factors was not possible for our review. Some intervention studies focused on the association of the infection control program and VAP prevention $[21,23,30]$ and NICU stay [23]. However, one possible explanation for this association could be the usage of humidifiers and closed-circuit ventilation in NICU which provide a major source for growth of microorganisms $[28,31]$. Hence, NICU environment itself can be a determining risk factor for development of VAP.

Risk factors that other studies have attributed to neonatal pneumonia of early onset but were absent from our review were antacid therapy [29], abnormal gastric aspirate, and low APGAR score among high-risk infants [32]. However, it has also been reported that often risk factors are absent in pneumonia of early onset, and that sudden onset of preterm labor by its very nature; is considered as an important risk factor [33].

Pneumonia is one of the leading causes of death among neonates in India. Thus, factors that affect neonatal mortality due to pneumonia and its occurrence are of great importance for any effort to improve child survival. However our review concludes that data and primary studies itself is negligible to substantiate a holistic view on factors associated with incidences and mortality of neonatal pneumonia. There is no 
conclusive evidence on risk factors of neonatal pneumonia other than ventilator associated pneumonia and hence it is recognized with this review that neonatal pneumonia, which comprises the majority of the burden of neonatal sepsis, continues to be an understudied issue in the Indian neonatal health scenario. To conclude, we can say that there is an emergent need to prioritize research toward generating evidence on neonatal pneumonia and determining factors for its development and mortality.

Acknowledgments: The authors would like to extend the gratitude to following persons for their guidance and support throughout the development process of this manuscript: Dr Manoj Das, Director Projects, The INCLEN Trust International, New Delhi; Dr Anju Sinha, Deputy Director General, Scientist 'E', Division of Child Health, Indian Council of Medical Research, New Delhi; Dr KK Diwakar, Professor and Head, Department of Neonatology, Associate Dean, Malankara Orthodox Syrian Church Medical College, Kerala; Mrs Ratheebhai V, Senior Librarian and Information Scientist, at Manipal School of Communication, Manipal Academy of Higher Education (MAHE), Manipal; Dr Ravinder M Pandey, Professor and Head, Department of Biostatistics, All India Institute of Medical Sciences, New Delhi; Dr B Shantharam Baliga, Professor, Department of Paediatrics, Kasturba Medical College, Mangalore, Karnataka; Dr Shrinivas Darak, Senior researcher, PRAYAS, Pune, Maharashtra; Dr Unnikrishnan B, Associate Dean and Professor, Department of Community Medicine, Kasturba Medical College, Mangalore. The authors would like to thank Dr. Ravishankar N, Assistant Professor, Department of Data Science, Prasanna School of Public Health (PSPH), MAHE, Manipal for conducting meta-analysis. Ethics clearance: Institutional Ethics Committee, Manipal Academy of Higher Education, Manipal; February 16, 2015.

Contributors: NSN: principal investigator for the project and guarantor for this article. He conceptualized the research idea and provided overall technical guidance; LESL: co-investigator for the project. conceptualized the research idea and provided overall technical guidance. In addition, LL helped in developing search terms; VSD: drafted the manuscript and contributed in drafting the full study report to the funder; SM: conducted the search, piloted the study selection process, drafted and piloted the data extraction form, selected studies, extracted data, performed risk of bias, synthesized data, and drafted the full study report to the funder; MAG: conducted the search, piloted the study selection process, drafted and piloted the data extraction form, selected studies, extracted data, performed risk of bias, synthesized data, and drafted the full study report to the funder; TL: conducted the search, piloted the study selection process, selected studies, conducted hand searching, extracted data, performed risk of bias, synthesized data, and drafted the full study report to the funder; BTV: administrative coordinator for the project, and conceptualized the research idea. She has also provided technical guidance throughout the project, during protocol development, finalizing the full report and addressing the project expert comments. All authors approved the final version of manuscript, and are accountable for all aspects related to the study.

Funding: This work was supported by Bill and Melinda Gates Foundation through The INCLEN Trust International (Grant number: OPP1084307). The funding source had no contribution in study design, implementation, collection and interpretation of data and report writing. Competing interests: None stated.

\section{REFERENCES}

1. Child Mortality Estimates: Global and regional child deaths by cause. Accessed April 04, 2019. Available from: http://data. unicef.org

2. Duke T. Neonatal pneumonia in developing countries. Arch Dis Child Fetal Neonatal Ed. 2005; 90:F211-FF219.

3. Ghimire M, Bhattacharya S, Narain J. Pneumonia in South-East Asia Region: Public health perspective. Indian J Med Res. 2012; 135:459.

4. Choudhury AM, Nargis S, Mollah AH, et al. Determination of risk factors of neonatal pneumonia. Mymensingh Med J. 2010;19:323-9.

5. Yang L, Zhang Y, Yu X, Luo M. Prevalence and risk factors of neonatal pneumonia in China: A longitudinal clinical study. Biomed Res. 2018; 29:57-60.

6. Almirall J, Serra-Prat M, Bolíbar I, et al. Risk factors for community-acquired pneumonia in adults: A systematic review of observational studies. Respiration 2017; 94:299-311.

7. Liu B, Li SQ, Zhang SM, et al. Risk factors of ventilatorassociated pneumonia in pediatric intensive care unit: A systematic review and meta-analysis. J Thorac Dis. 2013; 5:525-31.

8. Nair NS, Lewis LE, Godinho M, et al. Risk factors for neonatal pneumonia in India: A systematic review and meta-analysis. PROSPERO 2016 CRD42016044019. Available from:https:// www.crd.york.ac.uk/prospero/display_record.php? ID $=C R D$ 42016044019

9. Nair NS, Lewis LE, Lakiang T, et al. Risk factors for mortality due to neonatal pneumonia in India: A systematic review and meta-analysis. PROSPERO 2016 CRD 42016045398. Available from:https://www.crd.york.ac.uk/prospero/display_record.php? $I D=C R D 42016045398$

10. Nair NS, Lewis LE, Godinho M, et al. Factors associated with neonatal pneumonia in India: Protocol for a systematic review and planned meta-analysis. BMJ Open. 2018; 8: e018790.

11. Nair $S$, Lewis LE, Lakiang $T$, et al. Factors associated with mortality due to neonatal pneumonia in India: A protocol for systematic review and planned meta-analysis. BMJ Open. 2018;8:e18790.

12. Liberati A, Altman DG, Tetzlaff J, et al. The PRISMA statement for reporting systematic reviews and meta-analyses of studies that evaluate health care interventions: explanation and elaboration. Ann Intern Med. 2009;151:W65-94.

13. Stroup DF, Berlin JA, Morton SC, et al. Meta-analysis of observational studies in epidemiology: A proposal for reporting. JAMA. 2000; 283:2008-12.

14. Wong WC, Cheung CS, Hart GJ. Development of a quality assessment tool for systematic reviews of observational studies (QATSO) of HIV prevalence in men having sex with men and associated risk behaviours. Emerg Themes Epidemiol. 2008; $5: 23$.

15. Tripathi S, Malik GK, Jain A, et al. Study of ventilator associated pneumonia in neonatal intensive care unit: Characteristics, risk factors and outcome. Internet $\mathrm{J}$ Med Update. 2010; 5:12-19.

16. Mir ZH, Ali I, Qureshi OA, et al. Risk factors, pathogen profile and outcome of ventilator associated pneumonia in a neonatal intensive care unit. Int J Contemp Pediatr. 2015; 2:17-20.

17. Mathur NB, Garg K, Kumar S. Respiratory distress in neonates with special reference to pneumonia. Indian Pediatr. 2002; 39:529-37.

18. Schünemann HJ, Oxman AD, Higgins JP, et al. Presenting results and 'Summary of findings' tables. In: Higgins J, Green S, editors. 
Cochrane Handbook for Systematic Reviews of Interventions. Version 5.1.0. The Cochrane Collaboration, 2011. Available from: www.cochranehandbook.org

19. Thatrimontrichai A, Rujeerapaiboon N, Janjindamai W, et al. Outcomes and risk factors of ventilator-associated pneumonia in neonates. World J Pediatr. 2017; 13:328-34.

20. Lee PL, Lee WT, Chen HL. Ventilator-Associated Pneu-monia in Low Birth Weight Neonates at a Neonatal Intensive Care Unit: A Retrospective Observational Study. Pediatr Neonatol. 2017; 58:16-21.

21. Zhou Q, Lee SK, Jiang SY, et al. Efficacy of an infection control program in reducing ventilator-associated pneumonia in a Chinese neonatal intensive care unit. Am J Infect Control. 2013; 41:1059-64.

22. Apisarnthanarak A, Holzmann-Pazgal G, Hamvas A, et al. Ventilator-associated pneumonia in extremely preterm neonates in a neonatal intensive care unit: Characteristics, risk factors, and outcomes. Pediatrics. 2003; 112:1283-9.

23. Azab SF, Sherbiny HS, Saleh SH, et al. Reducing ventilatorassociated pneumonia in neonatal intensive care unit using "VAP prevention Bundle": A cohort study. BMC Infect Dis. 2015; $15: 314$.

24. Amin AJ, Malam PP, Asari PD, et al. Sensitivity and resistance pattern of antimicrobial agents used in cases of neonatal sepsis at a tertiary care center in western India. Int J Pharm Sci Res. 2016; 7:3060-67.

25. Islam MM, Hossain MM, Al Mamun MA, et al. Risk factors which affects the change of antibiotics in neonatal pneumonia observed in a tertiary care hospital. Northern Int Med Coll. 2014; 6:21-4.

26. Medeiros FD, Alves $\mathrm{VH}$, Valete $\mathrm{CO}$, et al. Invasive care procedures and neonatal sepsis in newborns with very low birth weights: A retrospective descriptive study. Online Braz. J Nurs. 2016; 15:704-12.

27. Afify M, AI-Zahrani S, Nouh MA. Risk Factors for the Development of Ventilator - Associated Pneumonia in Critically-ill Neonates. Life Sci J. 2012; 9:302-7.

28. Tan B, Zhang F, Zhang X, et al. Risk factors for ventilatorassociated pneumonia in the neonatal intensive care unit: a meta-analysis of observational studies. Eur J Pediatr. 2014; 173:427-34.

29. Afjeh SA, Sabzehei MK, Karimi A, et al. Surveillance of ventilator-associated pneumonia in a neonatal intensive care unit: Characteristics, risk factors, and outcome. Arch Iran Med. 2012; 15:567-71.

30. Ryan RM, Wilding GE, Wynn RJ, et al. Effect of enhanced ultraviolet germicidal irradiation in the heating ventilation and air conditioning system on ventilator-associated pneu-monia in a neonatal intensive care unit. J Perinatol. 2011; 31:607-14.

31. Garland JS. Strategies to prevent ventilator-associated pneumonia in neonates. Clin Perinatol. 2010; 37:629-43.

32. Mahendra I, Retayasa IW, Kardana IM. Risk of early onset pneumonia in neonates with abnormal gastric aspirate. Pediatrica Indonesiana. 2008;48:110-13.

33. Webber S, Wikinson AR, Lindsell D, et al. Neonatal pneu-monia. Arch Dis Child. 1990; 65:207-11.

\section{ERRATA}

Please note the following correction in the Clinical case letter titled "Early onset predominantly diffuse lung disease in an infant of combined methylmalonicacidemia with hyperhomocysteinemia cobalamin C type" published in Indian Pediatr. 2020;57:1079-1080. doi: $\quad 10.1007 / \mathrm{s} 13312-020-2045-\mathrm{x}$

The name of the authors should be 'Lvchang Zhu, Chanchan Hu, Sheng Ye, Chenmei Zhang' in place of 'Chenmei Zhang.' The affiliation of all authors is 'Department of Pediatric Intensive Care Unit,The Children's Hospital of Zhejiang University School of Medicine, 3333 Binsheng Road, Hangzhou, 310003, Zhejiang Province, China' and author C. Zhang is the corresponding author. Appropriate corrections have already been done in the web version at http://www.indianpediatrics.net/nov2020/1079.pdf on October $9,2021$.

Please note the following correction in the research paper titled "Pediatric inflammatory multisystem syndrome associated with SARS-CoV-2: A retrospective cohort study from Argentina" published in Indian Pediatr. 2021;58:639-642. doi: 10.1007/s13312021-2259-6.

The name of the fifth author should be 'Micaela Picollo' in place of 'Micela Picollo.' Additionally, her affiliation should be 'Department of Infectious Diseases, Hospital Juan P Garrahan Combate de los Pozos 1881, Argentina' in place of 'Department of Rheumatology, Hospital Juan P Garrahan Combate de los Pozos 1881, Argentina.' Appropriate corrections have already been done in the web version at http://www.indianpediatrics.net/ july2021/july-639-642.htm on October 4, 2021. 